\title{
LC3, an autophagosome marker, is highly expressed in gastrointestinal cancers
}

\author{
AKIKO YOSHIOKA $^{1}$, HIROSHI MIYATA ${ }^{1}$, YUICHIRO DOKI ${ }^{1}$, \\ MAKOTO YAMASAKI ${ }^{1}$, ITSURO SOHMA ${ }^{1}$, KUNIHITO GOTOH $^{1,2}$, SHUJI TAKIGUCHI ${ }^{1}$, \\ YOSHIYUKI FUJIWARA ${ }^{1}$, YASUO UCHIYAMA ${ }^{2}$ and MORITO MONDEN ${ }^{1}$ \\ Departments of ${ }^{1}$ Gastroenterological Surgery, and ${ }^{2}$ Cell Biology and Neuroscience, \\ Graduate School of Medicine, Osaka University, Osaka, Japan
}

Received February 26, 2008; Accepted April 7, 2008

DOI: 10.3892/ijo_00000028

\begin{abstract}
Autophagy is a bulk protein and organelle degradation process essential for cell maintenance and viability. Microtubule-associated protein 1 light chain 3 (LC3), the mammalian homologue of yeast Atg8, is involved in autophagosome formation during autophagy. The aim of this study was to investigate LC3 expression in gastrointestinal cancers to elucidate the role of autophagy in human cancer development. We evaluated LC3 expression by immunohistochemistry in 163 gastrointestinal cancers including 106 esophageal, 38 gastric and 19 colorectal cancers. Seventy precancerous intraepithelial neoplasias were found in esophageal cancer specimens. LC3 expression was compared with Ki-67 staining and expression of carbonic anhydrase (CA) IX, a hypoxic marker. LC3 was expressed in the cytoplasm of cancer cells, but not in noncancerous epithelial cells. A high expression of LC3 was observed in 53\% of esophageal, $58 \%$ of gastric and $63 \%$ of colorectal cancers. LC3 immunoreactive score gradually increased during early esophageal carcinogenesis in low- and high-grade intraepithelial neoplasia and T1 carcinoma, while it did not change in later cancer progression (T2-T4 carcinomas). In early esophageal carcinogenesis, LC3 expression correlated with Ki-67 labeling index $(\mathrm{p}=0.0001)$, but showed no significant association with CAIX expression. In esophageal cancers, LC3 expression did not correlate with various clinicopathological factors, including survival. LC3 is upregulated in various gastrointestinal cancers and partly associated with Ki-67 index. Our results suggest that LC3 expression is advantageous to cancer development especially in earlyphase carcinogenesis.
\end{abstract}

Correspondence to: Dr Hiroshi Miyata, Department of Gastroenterological Surgery, Graduate School of Medicine, Osaka University, 2-2 Yamadaoka, Suita, Osaka 565-0871, Japan

E-mail: hmiyata@surg2.med.osaka-u.ac.jp

Key words: light chain 3, autophagy, cancer development, gastrointestinal cancer, esophageal cancer

\section{Introduction}

Autophagy is an evolutionarily conserved process in which intracellular membrane structures sequester proteins and organelles to degrade these materials, and serves to eliminate defective cellular constituents, including subcellular structures such as mitochondria, to maintain cellular homeostasis (1-3). During autophagy, cytoplasmic components are engulfed by double-membrane structures known as autophagosomes, which fuse to lysosomal vesicles where the contents are degraded into their components (1). Autophagy also plays an important role as a self-eating system to produce energy for adaptation and survival under starvation, and is known to protect cells against stresses $(4,5)$. Autophagy is regulated by mammalian target of rapamycin (mTOR), which is a serine/threonine protein kinase that acts as a central regulator of cell growth and survival, through the action of class I phosphatidylinositol 3-kinase (PI3K) (6). Class I PI3K activates Akt1, which is another serine/threonine protein kinase located downstream of class I PI3K, and stimulates mTOR, leading to suppression of autophagy $(7,8)$.

So far, more than 20 genes have been identified as autophagy-related genes (ATG) in yeast $(9,10)$. Many of the yeast ATG genes are conserved in mammals, and many mammalian homologues have been identified and characterized $(11,12)$. Microtubule-associated protein 1 light chain 3 (LC3) is an autophagosomal orthologue of yeast Atg8, with approximately $30 \%$ amino acid homology with Atg8 $(11,13)$. It exists in two forms, LC3-I and its proteolytic derivative LC3-II (molecular weight, 18 and $16 \mathrm{kD}$, respectively) (14). LC3-I is localized in the cytoplasm, whereas LC3-II binds to autophagosomes (14). Introduction of autophagy by various stresses such as starvation, stimulates the conversion of LC3-I to LC3-II, and upregulation of LC3 expression (15). Thus, LC3 is a specific marker of autophagosome formation.

Recently, the role of autophagy in cancer development and cancer treatment has been investigated both in vitro and in vivo. There is ample evidence to suggest that autophagy is involved in tumor suppressor pathways. Inactivation of autophagy-specific genes, such as mammalian Atg6 homologue, Beclin 1, results in increased tumorigenesis in mice, and enforcement of the expression of such genes inhibits the formation of human breast tumors in mouse models (16). 
Furthermore, several cancer types, such as breast, ovarian and prostate, have a high frequency of allelic loss of Beclin 1 (17). On the other hand, other studies demonstrated that autophagy promotes tumor progression and protects some cancer cells against anticancer treatments. As tumors grow, cancer cells may require autophagy to survive nutrient-limiting and lowoxygen conditions, especially in the central area of the tumors, which is often poorly vascularized (18-21). Chloroquine, which is regarded as an autophagy inhibitor, enhances the animal's response to tamoxifen (22). Furthermore, autophagy protects some cancer cells against ionizing radiation (23). Thus, the role of autophagy in tumor suppression or development and tumor response to anticancer therapy is still unclear.

In the present study, we examined LC3 expression in gastrointestinal cancers including esophageal cancer, gastric cancer and colorectal cancer by immunohistochemistry. To our knowledge, this is the first study in which the role of autophagy in human gastrointestinal cancer tissues was investigated by immunohistochemistry. In addition, we investigated LC3 expression in multistage carcinogenesis of esophageal cancers and the relation between its expression and clinicopathological factors of those cancers. We then attempted to elucidate the role of autophagy in human cancer development.

\section{Materials and methods}

Patients and samples. Our study was conducted with 106 patients with esophageal squamous cell carcinoma (ESCC), 38 patients with gastric adenocarcinoma, and 19 patients with colorectal adenocarcinoma, who were admitted to our hospital between 1999 and 2004. They underwent curative resection at the Department of Gastroenterological Surgery, Osaka University Hospital. None had received pre-operative anticancer therapy. The tumors were classified according to the International Union against Cancer (UICC) guidelines and the criteria defined by the WHO International Histological Classification of Tumors (24). Whole surgically removed esophagus was histologically investigated with longitudinally cut 5-mm step sections, and we found 39 lesions of low-grade and 31 lesions of high-grade intraepithelial neoplasia in the 106 esophageal cancer patients. Low-grade intraepithelial neoplasia (LGIN) was defined as the presence of an atypical cell zone with large hyperchromatic nuclei and increased mitotic activity forming less than half of the stratified squamous epithelium. High-grade intraepithelial neoplasia (HGIN) referred to tumors with an atypical cell zone encompassing more than half of the epithelium. The study protocol was approved by the Human Ethics Review Committee of the Graduate School of Medicine, Osaka University and a signed consent form was obtained from each subject.

Immunohistological staining procedures and evaluation of staining. Surgical specimens were fixed in $10 \%$ formaldehyde and routinely processed for paraffin embedding. Histological sections (4 $\mu \mathrm{m}$ thick) were stained with hematoxylin and eosin. A representative section from each cancer with the deepest tumor infiltration and all sections of intraepithelial neoplasia of the esophagus were subjected to immunohistochemical examination.
For immunohistochemical analysis, we used LC3 rabbit polyclonal antibody (kindly provided by Dr Y. Uchiyama; dilution 1:200) (25), Ki-67 (mouse monoclonal antibody MIB-1; Dako, Carpinteria, CA; dilution 1:400), and carbonic anhydrase (CA) IX (N19) (goat polyclonal antibody, Santa Cruz, CA; dilution 1:200) on semi-serial sections of paraffinembedded, formalin-fixed material. Immunostaining was performed using streptavidin-peroxidase complex method as described previously (26). Briefly, $4-\mu \mathrm{m}$ paraffin sections were deparaffinized, endogenous peroxidase was blocked with $0.3 \% \mathrm{H}_{2} \mathrm{O}_{2}$ in methanol, and the sections were then covered with $10 \%$ normal rabbit serum for $20 \mathrm{~min}$ at room temperature. The sections were incubated $48 \mathrm{~h}$ at $4^{\circ} \mathrm{C}$ with primary antibodies, and then processed by the biotinstreptavidin method using a commercially-available kit, according to the instructions provided by the manufacturer (Vector Laboratories, Burlingame, CA). For negative controls, non-immunized mouse or rabbit IgG serum (Vector Laboratories, Burlingame, CA) was used as the primary antibody, which yielded negative results. Color was developed with diaminobenzidine tetrahydrochloride supplemented with $0.02 \%$ hydrogen peroxide, and the nuclei were counterstained with Meyer's hematoxylin. An antibody preincubated with antigen peptide was used for negative control tests, which yielded a uniformly negative result.

Evaluation of immunostaining. The immunoreactivity of LC3 was evaluated according to the intensity and percentage of positively stained cells. The percentage of positively stained cells was graded as follows: grade 0, 0-39\%; grade 1, 40-69\%, grade $2,>70 \%$. Immunostaining intensity was rated as follows: 0 , negative; 1 , weak; and 2 , strong. In addition, an immunoreactive score was calculated by the addition of the percentage score of positively stained cells and the score of staining intensity (0-4). Tumors with an immunoreactive score of 0-1 were designated 'low', and tumors with an immunoreactive score of 2-4 were designated 'high'. The Ki-67 index was calculated as a percentage of positive nuclei in the whole tumor section. Strong cell membranous CAIX expression was evaluated as positive staining. The percentage of CAIXpositive tumor cells in the whole tumor section was determined. Immunostaining was evaluated twice by two pathologists (A.Y. and H.M.) blinded to patient outcome and other clinical findings.

Western blot analysis. Western blot analysis was performed, as described previously (27). Total protein (35 $\mu \mathrm{g})$ from the tissues was used in this assay, and ischemic rat liver tissues were used as positive controls (25). Anti-human LC3 rabbit polyclonal antibody was used at a concentration of $1.0 \mu \mathrm{l} / \mathrm{ml}$. Detection of the protein bands was performed using the Amersham ECL detection system (Amersham, Arlington Heights, IL) according to the instructions provide by the manufacturer.

Statistical analysis. The Spearman's rank test was used to analyze the progressive increase in LC 3 expression in multistage squamous carcinogenesis. Differences in Ki-67 index and CAIX expression according to LC3 score were evaluated using Student's t-test. The association between 


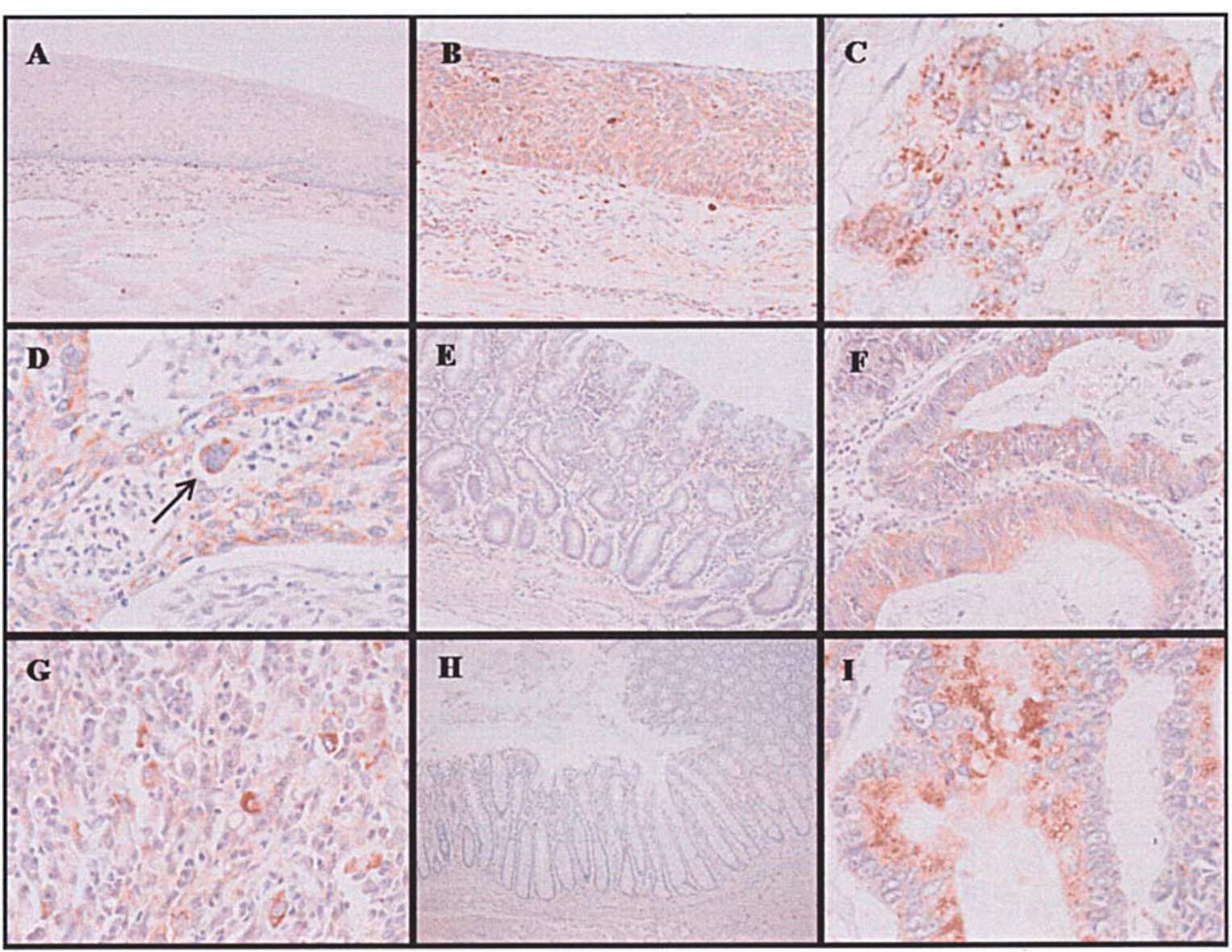

Figure 1. LC3 expression in cancerous and noncancerous gastrointestinal tissues. Immunohistochemical analysis of LC3 expression in esophageal tissues (A-D), gastric tissues (E-G) and colorectal tissues (H and I). Histopathological classification was noncancerous epithelium (A, E and H), esophageal highgrade intraepithelial neoplasia (B) and cancers (C, D, F, G and I). LC3 expression was not observed in the noncancerous epithelia, but in the cytoplasm of esophageal intraepithelial neoplasias and gastrointestinal cancer cells. Arrow, LC3 expression in the cytoplasm of cell with swollen or split nucleus. Magnification: (H) x40; (A and E) x100; (B, F and I) x200; (C, D and G) x400.

expression measured by immunohistochemistry and clinicopathological parameters was analyzed using Chi-square test and Student's t-test, Actual survival was calculated by the Kaplan-Meier and statistically evaluated by the log-rank test. In all analyses, $\mathrm{p}<0.05$ was accepted as statistically significant. These analyses were carried out using Stat View J5.0 software package (Abacus Concepts, Inc., Berkeley, CA).

\section{Results}

LC3 expression in noncancerous epithelial cells. In the noncancerous esophageal epithelium without significant inflammation, no immunostaining with LC3 antibody was observed in stratified squamous epithelial cells, including granular, keratinizing, basal and parabasal layers (Fig. 1A). In the lamina propria, some infiltrating mononuclear cells were strongly positive for LC3 expression in the cytoplasm. Some of these cells, such as macrophages, were also immunopositive for CD68 expression (data not shown). As for the esophageal epithelial cells, LC3 expression was not detected in columnar epithelia of noncancerous gastric and colorectal mucosa (Fig. 1E and 1H).

LC3 expression in gastrointestinal cancers. In contrast to noncancerous epithelial cells, LC3 expression was observed in the cytoplasm of gastrointestinal cancers including esophageal, gastric and colorectal cancers (Fig. 1C, D, F, G and I). In many cases, LC3 expression showed dot-like staining in the cytoplasm of cancer cells, whereas it was not detected in the nuclei of cancer cells. In general, LC3 was highly expressed in cancer cells located at the front of cancer nests rather than cancer cells in the central area of cancer nests. However, a few cancer cells in the central area of cancer nests showed LC3 strong expression (Fig. 1D, Fig. 4A and C). High expression of LC3 was observed in 53\% of esophageal cancers (56 of 106 cases), 58\% of gastric cancers (22 of 38 cases) and $63 \%$ of colorectal cancers (12 of 19 cases).

Western blot analysis. To confirm the specificity of LC3 antibody, a limited set of tissue samples (four matched noncancerous mucosae and ESCC tissues) were subjected to Western blot analysis. As shown in Fig. 2, protein bands immunopositive for LC3-I and -II forms were clearly evident in each sample. The liver of a rat that had been kept in a starvation condition was used as a positive control (lane 9). Noncancerous esophageal tissues generally yielded no, or only a faint, band for LC3-I and -II (lanes 1, 3, 5, 7). All tumor tissues yielded stronger bands for LC3, especially for LC3-II, compared with noncancerous mucosae (lanes 2, 4, 6, 8). 


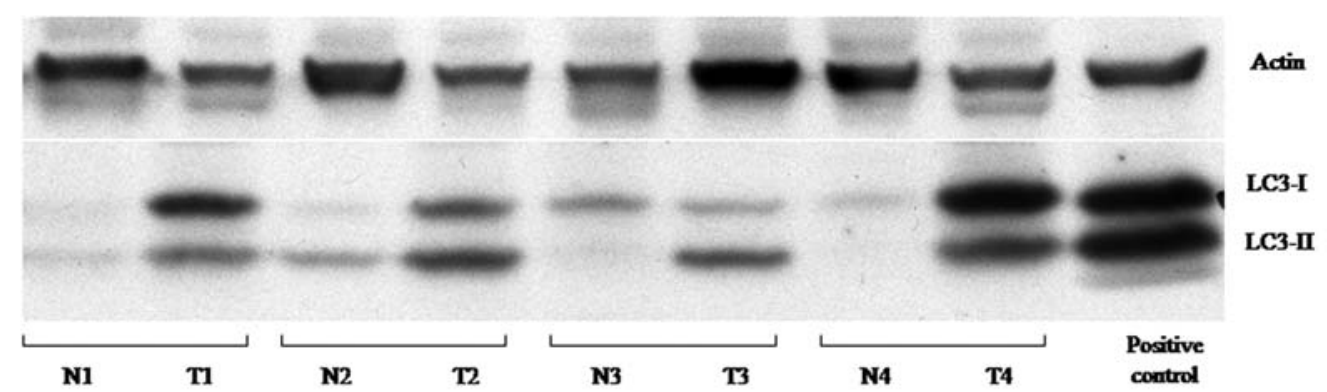

LC3-I : 18kD

LC3-II: $16 \mathrm{kD}$

Figure 2. Western blot analysis of LC3 in noncancerous esophageal mucosa and esophageal cancer tissues. Proteins extracted from representative noncancerous esophageal mucosa (lanes 1, 3, 5, 7) and esophageal cancer tissues (lanes 2, 4, 6, 8) were subjected to Western blot analysis. Tumor samples displayed both prominent bands for LC3-I located at $18 \mathrm{kD}$ and for LC3-II located at $16 \mathrm{kD}$, while noncancerous esophageal tissues generally yielded no, or only a faint, band for LC3-I and LC3-II. A rat liver tissue served as a positive control (lane 9). N, noncancerous esophageal mucosa; T, esophageal cancer tissues.

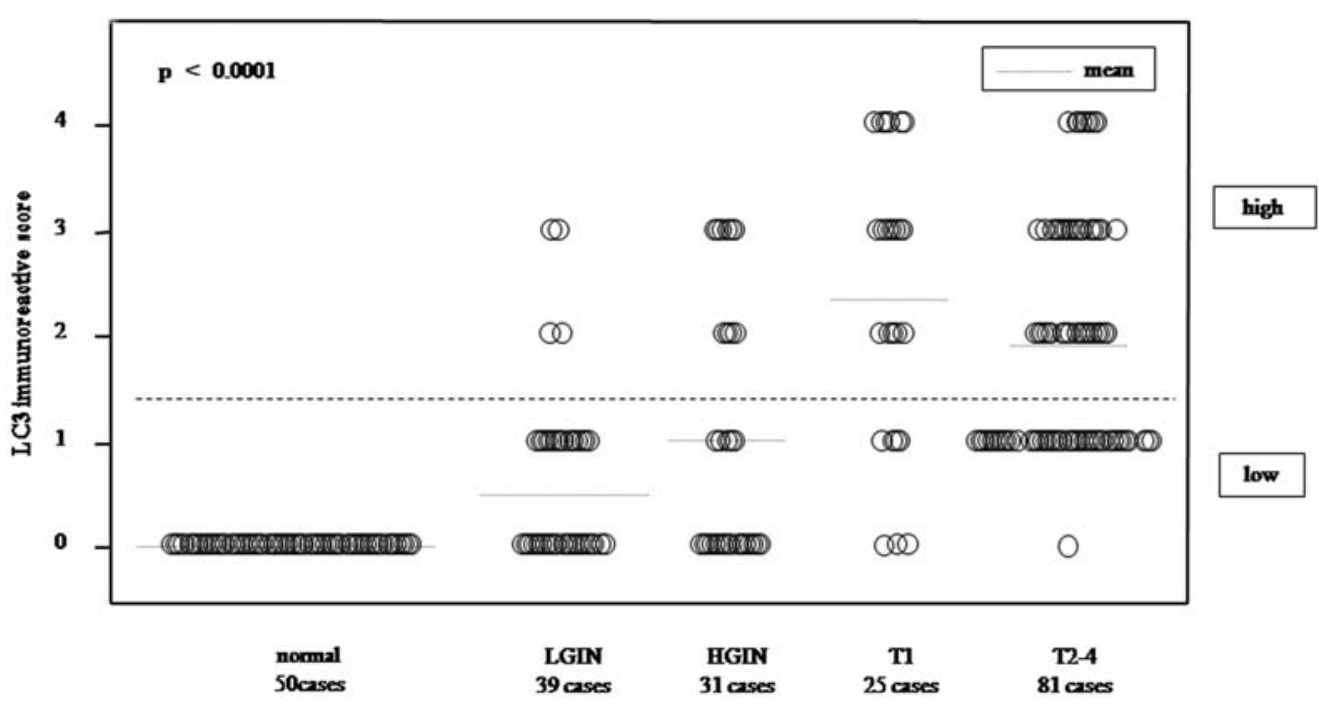

Figure 3. LC3 immunoreactive scores for different esophageal lesions. LC3 scores for individual esophageal lesions (open circles) along with the average for the group. The LC3 score increased progressively from noncancerous epithelia to esophageal squamous cell carcinomas. (p<0.0001; Spearman's rank test). LGIN, low-grade intraepithelial neoplasia; HGIN, high-grade intraepithelial neoplasia.

LC3 expression in esophageal intraepithelial neoplasias and carcinomas. We examined LC3 expression in multistage carcinogenesis in ESCC. Similar to advanced cancers, cytoplasmic LC3 expression was noted in early cancers and intraepithelial neoplasias (IN) (Fig. 1B). LC3 immunoreactive scores were determined in all esophageal specimens. While high LC3 expression was not observed in noncancerous epithelial cells and was observed in only $10 \%$ (4 of 39 cases) of LGINs, LC3 overexpression was observed in 32\% (10 of 31 cases) of HGINs and in 56\% (59 of 106 cases) of ESCCs (Fig. 3). There was a significant increase in LC3 immunoreactive score from noncancerous epithelium to ESCCs. However, there was no significant difference in LC3 score between early ESCCs (T1) and advanced ESCCs (T2T4) (Fig. 3).

To investigate the possible effect of LC3 on cell proliferation, we determined the index of $\mathrm{Ki}-67$, which is a cell cycle-related molecule, for all of the esophageal specimens.
In general, LC3-overexpressing tumor cells tended to show positive Ki-67 staining (Fig. 4A and B). In esophageal INs and early ESCCs (T1), Ki-67 index was significantly higher in LC3-overexpressing tumors than low LC3-expression tumors $(\mathrm{p}<0.0001)$ (Fig. 5A). On the other hand, in advanced ESCCs (T2-T4), there was no significant difference in Ki-67 index between high- and low-LC3 expressing tumors $(\mathrm{p}=0.4049)$ (Fig. 5B). Autophagy is induced under stress conditions such as hypoxia. Accordingly, we investigated the relationship between LC3 expression and CAIX expression, which is regarded as a marker of tissue hypoxia (28). In ESCCs, the expression of LC3 was opposite that of CAIX; CAIX expression was generally evident in the central area of cancer nests including focal necrosis, while LC3 was highly expressed in the front area of cancer nests as described above (Fig. 4C and D). In esophageal INs and early ESCCs (T1), CAIX index was quite low irrespective of LC3 expression. In advanced ESCCs (T2-T4), CAIX index was higher than in 


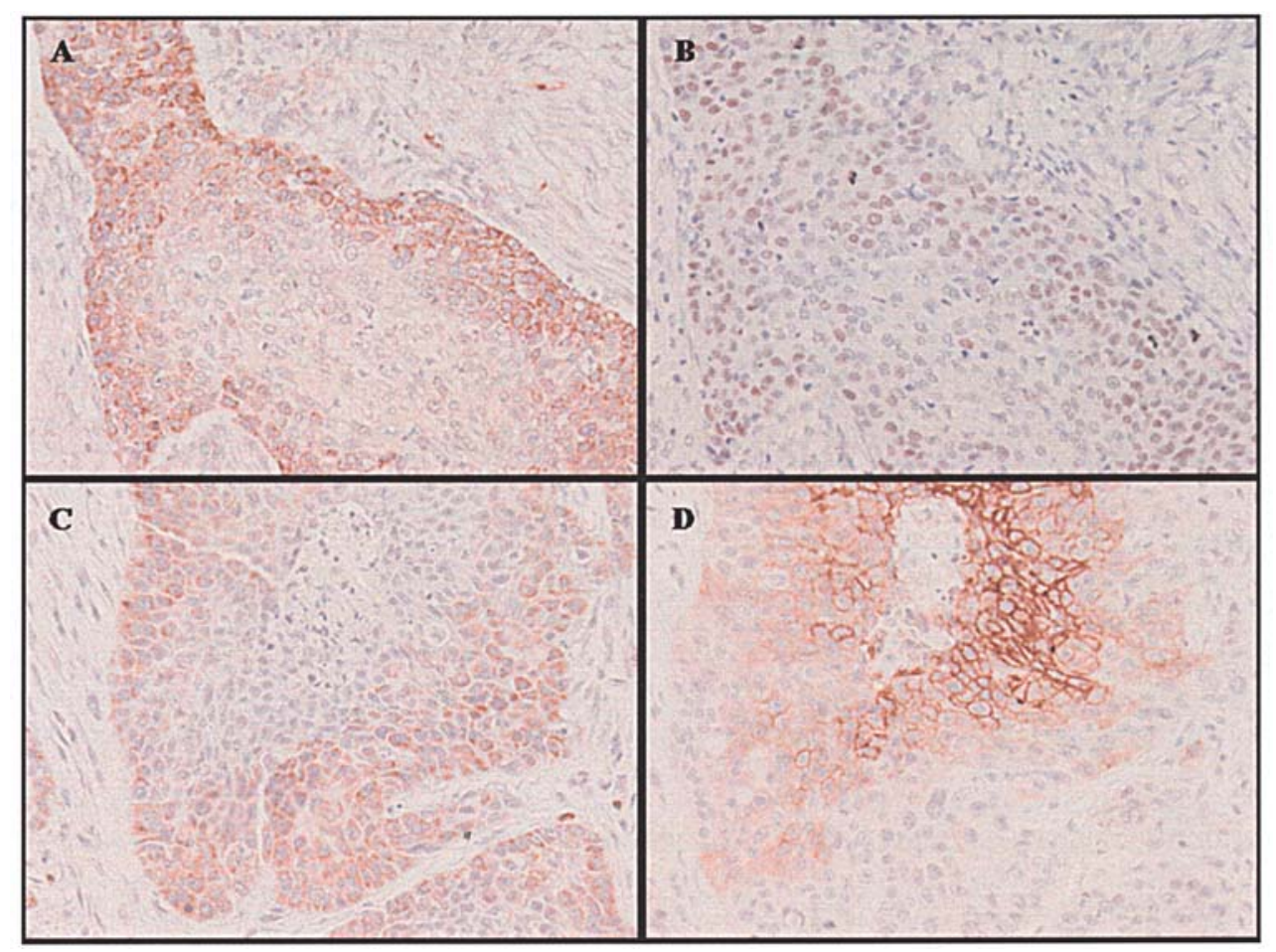

Figure 4. Comparison of LC3 expression with Ki-67 and CAIX expression in esophageal cancers. LC3-immunostained sections (A and C) with consecutive sections of Ki-67 (B) and CAIX (D) immunostaining in esophageal cancers. LC3 was mostly expressed around the lateral area of cancer nest, where Ki-67positive cells were frequently observed. On the other hand, CAIX was frequently expressed in the center of the cancer nest. Magnification, x200.
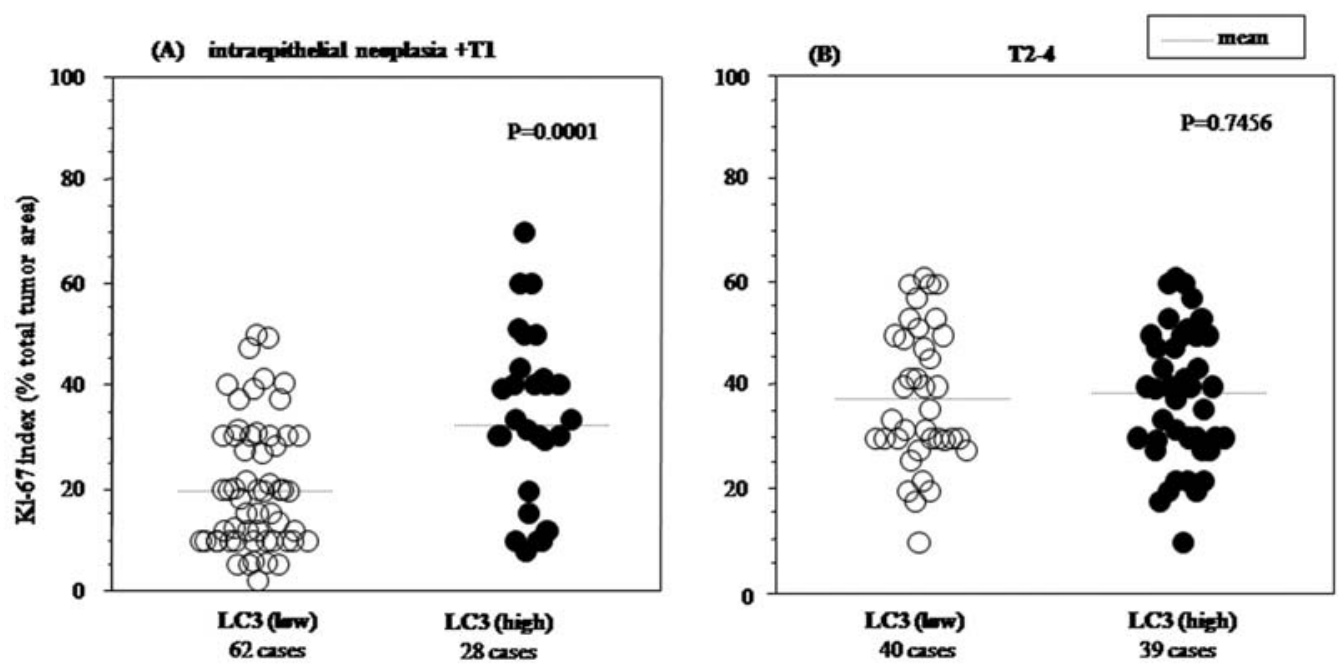

Figure 5. Relationship between Ki-67 index and LC3 score in different stages of esophageal carcinogenesis. Ki-67 index for esophageal lesions with low LC3 score (open circles) and high LC3 score (closed circles) in early carcinogenesis (A), including intraepithelial neoplasias and T1 cancers, and advanced (T2-T4) cancers (B). The average Ki-67 index for each group is indicated by the horizontal line. A significant difference in Ki-67 index between LC3 highand low-score was found in early carcinogenesis $(\mathrm{p}=0.0001)$ but not in advanced cancers. $(\mathrm{p}=0.7456)$. P-value was evaluated with Student's $\mathrm{t}$-test.

INs and superficial cancers, but there was no significant correlation between LC3 expression and CAIX index (data not shown).

Correlation between LC3 expression in ESCCs and clinicopathological parameters. To investigate the effect of LC3 expression on tumor aggressiveness, we examined the relationship between LC3 expression and clinicopathological parameters, including age, gender, differentiation, tumor depth, lymph node metastasis and tumor stage. However, we did not find a significant association between LC3 expression and the various clinicopathological parameters (Table I). In addition, no significant association was found between LC3 expression and overall survival rate (Fig. 6).

\section{Discussion}

In the present study, we examined LC3 expression in gastrointestinal cancers including esophageal, gastric and colorectal cancers, and found that LC3 is highly expressed in many 


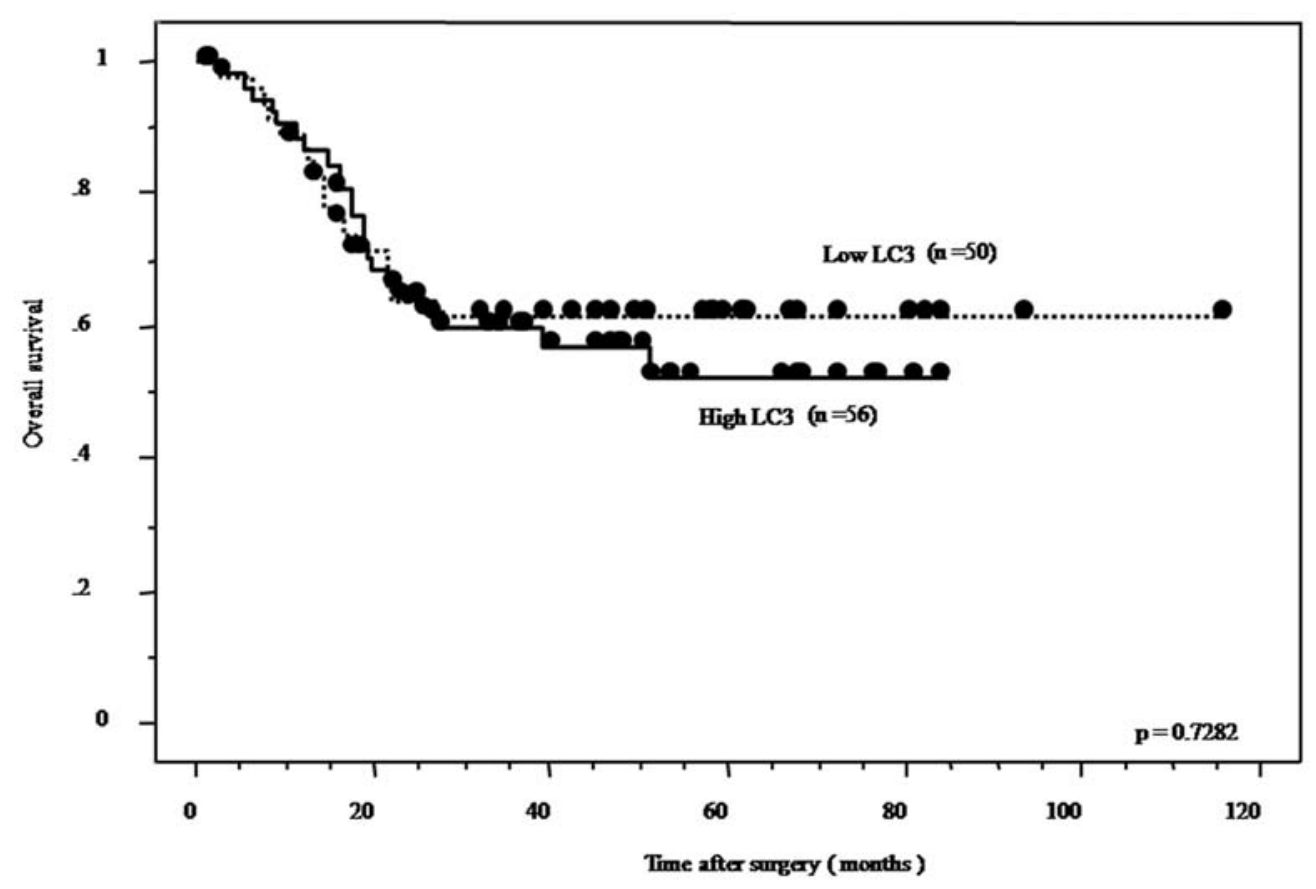

Figure 6. Post-operative survival of patients with esophageal cancer according to LC3 expression. Survival curves of esophageal cancer patients with highand low-LC3 score were plotted by Kaplan-Meier method and their difference was evaluated by the log-rank test. Survival was measured from the date of surgery to the date of the last follow-up or death. There was no significant difference in overall survival ratio between the two groups.

gastrointestinal cancer cells, compared with noncancerous epithelial cells. It has been recognized that the standard method for assessing autophagic activity is the demonstration of autophagic vesicles by electron microscopy $(14,29,30)$. However, electron microscopy is not suitable for assessing whole tumor samples, and it is sometimes difficult to distinguish autophagic vacuoles from other structures just by morphology using electron microscopy (31). Another method of monitoring autophagy in mammals is LC3-based assay. When autophagy occurs, conversion of a fraction of cytosolic form LC3-I to autophagic membrane form LC3-II can be detected by immunoblotting of LC3 proteins. This change in intracellular localization of LC3 protein is considered to correlate with autophagic activity (14), but there may be a problem with a limited capacity to detect LC3 proteins by immunoblotting in human tissues (31). On the other hand, the immunohistochemical method of LC3 antibody used in this study is suitable for evaluating many samples of human cancer. LC3 expression by immunohistochemistry represents the steady-state LC3 level including both LC3-I and LC3-II as shown by Western blot analysis, but the amount of LC3-II is considered to correlate well with the formation of autophagosomes because not LC3-I but LC3-II binds and localizes to autophagosomes (14). Thus, while this immunohistochemical method is not always specific for monitoring autophagy, LC3 expression by immunohistochemistry can be a good indicator of autophagic activity in noncancerous epithelial cells and cancer cells.

Increasing evidence indicates that autophagy has an important role in cancer development. However, it is not clear whether autophagy suppresses tumor development or provides cancer cells with a rescue mechanism under stress conditions such as starvation, promoting tumor development (32-36). Previous studies demonstrated that baseline house- keeping levels of autophagy occur in most noncancerous cells to prevent accumulation of protein aggregates and defective cellular substructures, and that it is upregulated when those cells encounter stress conditions such as starvation $(1,10,21,37,38)$. However, LC3-negativity in noncancerous cells in this study indicates that baseline levels of autophagy in human gastrointestinal noncancerous tissues under normal conditions are so low that they cannot be detected by immunohistochemistry. In contrast, LC3 expression in gastrointestinal cancers suggests that induction of autophagy is advantageous to tumorigenesis in gastrointestinal cancers. One supporting result is that there was a tendency for LC3-overexpressing cancer cells to exhibit positive Ki-67 nuclear staining in the same tumor. Another supporting result was the positive correlation between Ki-67 index and LC3 expression in esophageal INs and early ESCCs, but not in advanced cancers. Thus, the role of autophagy in cancer development may be more important in an early stage of carcinogenesis than in advanced cancers. According to our unpublished data of immunohistochemistry, mTOR, which suppresses autophagy, was overexpressed in human ESCCs, compared with noncancerous tissues, and its high expression was more frequently observed in advanced ESCCs than in early ESCCs. Collectively, the reason for the lack of correlation between LC3 expression and Ki-67 index in advanced cancer may be related to downregulation of autophagy induced by mTOR in advanced cancers, compared with early cancers.

Thus far, the main role of autophagy has been considered to adapt to metabolic stress such as starvation and hypoxia, and in some cases, autophagy has also been regarded as a form of programmed cell death $(39,40)$. In each case, theoretically, autophagy should occur in cancer cells located in the central area of cancer nests. Indeed, in our study, there were some LC3-overexpressing cancer cells in the central 
Table I. Relationship between various clinicopathological parameters and LC3 expression in 106 ESCC patients.

\begin{tabular}{|c|c|c|c|}
\hline & $\begin{array}{l}\text { Low LC3 expression }(\mathrm{n}=50) \\
\mathrm{n}(\%)\end{array}$ & $\begin{array}{l}\text { High LC3 expression }(\mathrm{n}=56) \\
\mathrm{n}(\%)\end{array}$ & P-value ${ }^{a}$ \\
\hline Age (years) & $62 \pm 8.03$ & $64 \pm 7.57$ & $\mathrm{p}=0.3254^{\mathrm{b}}$ \\
\hline \multicolumn{4}{|l|}{ Gender } \\
\hline Male & 49 (49) & $52(51)$ & \\
\hline Female & $1(20)$ & $4(80)$ & $\mathrm{p}=0.2646$ \\
\hline \multicolumn{4}{|l|}{ Location } \\
\hline Upper thoracic & $4(40)$ & $6(60)$ & \\
\hline Middle thoracic & $18(37)$ & $31(63)$ & \\
\hline Lower thoracic & $28(60)$ & $19(40)$ & $\mathrm{p}=0.0743$ \\
\hline \multicolumn{4}{|l|}{ Depth of tumor invasion ${ }^{c}$} \\
\hline $\mathrm{pT} 1+2$ & $21(48)$ & $23(52)$ & \\
\hline pT3+4 & $29(47)$ & $33(53)$ & $\mathrm{p}=0.9232$ \\
\hline \multicolumn{4}{|l|}{ Lymph node metastasis ${ }^{c}$} \\
\hline pNO & $11(33)$ & $22(67)$ & \\
\hline pN1 & $39(53)$ & $34(47)$ & $\mathrm{p}=0.0562$ \\
\hline \multicolumn{4}{|l|}{ Differentiation (SCC) } \\
\hline Well differentiated & $12(39)$ & $19(61)$ & \\
\hline Moderately differentiated & $24(53)$ & $21(47)$ & \\
\hline Poorly differentiated & $14(47)$ & $16(53)$ & $\mathrm{p}=0.4574$ \\
\hline \multicolumn{4}{|l|}{ UICC p-Stage ${ }^{c}$} \\
\hline $\mathrm{I}+\mathrm{II}$ & $19(38)$ & $31(62)$ & \\
\hline III+IV & $31(55)$ & $25(45)$ & $\mathrm{p}=0.0753$ \\
\hline \multicolumn{4}{|l|}{ Lymphatic invasion } \\
\hline Ly(-) & $9(38)$ & $15(62)$ & \\
\hline $\operatorname{Ly}(+)$ & $41(50)$ & $41(50)$ & $\mathrm{p}=0.2829$ \\
\hline \multicolumn{4}{|l|}{ Vascular invasion } \\
\hline $\mathrm{V}(-)$ & $30(46)$ & $35(54)$ & \\
\hline $\mathrm{V}(+)$ & $20(49)$ & $21(51)$ & $\mathrm{p}=0.7929$ \\
\hline
\end{tabular}

${ }^{\mathrm{a}}$ Chi-square test, ${ }^{\mathrm{b}}$ Student's t-test. ${ }^{\mathrm{C}}$ Based on TNM classification system of the International Union against Cancer (UICC). SCC, squamous cell carcinoma.

area of cancer nests, but such cells were only a few among the LC3-immunopositive cells and did not affect evaluation of LC3 expression in whole cancer tissues. Unfortunately, in the current study, we could not determine the fate of those cells, whether they survive or undergo cell death. In general, LC3 high expression was observed in the front of cancer nests, in contrast to the high expression of CAIX in the central area of cancer nests corresponding to the hypoxic area. Previous research demonstrated that autophagy is essential for maintaining cellular bioenegenetics during growth factordeprivation, that autophagy-dependent ATP production promotes cell survival, and that autophagy also contributes to the turn-over of cytoplasmic cellular components (35). Thus, another possible role of autophagy in cancer may be to produce cellular components and energy for actively proliferating cells in rapidly growing tumor areas, such as the front area of cancer nests.
Apoptosis and autophagy, which are both self-destructive processes, have long been classified as a unique form of programmed cell death, apoptotic cell death as type I and autophagic cell death as type II cell death $(39,41)$. However, recent evidence demonstrates that autophagy and apoptosis are regulated by common upstream signals and share common components, such as Bcl-2 family members, death associated protein kinase (DAPK) family and PI3K/Akt signaling pathway, and that there is a functional relationship between autophagy and apoptosis (42). A recent study showed that autophagy neither delays nor promotes apoptosis, but it is required for apoptotic cells to generate the signal for engulfment during embryonic development (43). Regarding cancer development and treatment, autophagy and apoptosis commonly occur in the same cells (20). In some cases, inhibition of apoptosis causes autophagy (20), while in other cases, inhibition of autophagy triggers apoptosis (22). Thus, 
targeting autophagy may be a useful strategy for cancer treatment. When autophagy is used for cancer cells to adapt to stress conditions such as metabolic stress and cytotoxic therapy, inhibition of autophagy may result in increased cell death. Alternatively, against cancer cells that have a deficit in apoptotic machinery, induction of massive autophagy may lead to the death of these cells. Whether autophagy should be turned on or off for cancer therapy may vary depending on the biological context. In this study, unexpectedly, we did not find a significant relationship between LC3 expression and tumor aggressiveness such as tumor depth and lymph node metastasis, and also no relationship between LC3 expression and prognosis of patients with ESCCs. In order to utilize the modulation of autophagy for cancer therapy, further investigation into the role of autophagy in human cancers is needed.

In conclusion, we demonstrated in the present study, upregulation of LC3, a marker of autophagic activity, in various gastrointestinal cancers, and its expression was positively correlated with Ki-67 index in early cancers. Our findings suggest that LC3 expression is advantageous to cancer development in early phases of carcinogenesis.

\section{References}

1. Klionsky DJ and Emr SD: Autophagy as a regulated pathway of cellular degradation. Science 290: 1717-1721, 2000.

2. Ohsumi Y: Molecular dissection of autophagy: two ubiquitinlike systems. Nat Rev Mol Cell Biol 2: 211-216,2000.

3. Elmore SP, Qian T, Grissom SF and Lemasters JJ: The mitochondrial permeability transition initiates autophagy in rat hepatocytes. FASEB J 15: 2286-2287, 2001.

4. Munafo DB and Colombo MI: A novel assay to study autophagy: regulation of autophagosome vacuole size by amino acid deprivation. J Cell Sci 114: 3619-3629, 2001.

5. Meijer AJ and Dubbelhuis PF: Amino acid signalling and the integration of metabolism. Biochem Biophys Res Commun 313: 397-403, 2004.

6. Rusten TE, Lindmo K, Juhasz G, Sass M, Seglen PO, Brech A and Stenmark H: Programmed autophagy in the Drosophila fat body is induced by ecdysone through regulation of the PI3K pathway. Dev Cell 7: 179-192, 2004.

7. Petiot A, Ogier-Denis E, Blommaart EF, Meijer AJ and Codogno P: Distinct classes of phosphatidylinositol 3'-kinases are involved in signaling pathways that control macroautophagy in HT-29 cells. J Biol Chem 275: 992-998, 2000.

8. Meijer AJ and Codogno P: Regulation and role of autophagy in mammalian cells. Int J Biochem Cell Biol 36: 2445-2462, 2004.

9. Klionsky DJ, Cregg JM, Dunn WA Jr, et al: A unified nomenclature for yeast autophagy-related genes. Dev Cell 5: 539-545, 2003.

10. Tsukada M and Ohsumi Y: Isolation and characterization of autophagy-defective mutants of Saccharomyces cerevisiae. FEBS Lett 333: 169-174, 1993.

11. Tanida I, Ueno T and Kominami E: LC3 conjugation system in mammalian autophagy. Int J Biochem Cell Biol 36: 2503-2518, 2004.

12. Klionsky DJ, Cuervo AM and Seglen PO: Methods for monitoring autophagy from yeast to human. Autophagy 3: 181-206, 2007.

13. Hemelaar J, Lelyveld VS, Kessler BM and Ploegh HL: A single protease, Apg4B, is specific for the autophagy-related ubiquitinlike proteins GATE-16, MAP1-LC3, GABARAP, and Apg8L. J Biol Chem 278: 51841-51850, 2003.

14. Kabeya Y, Mizushima N, Ueno T, et al: LC3, a mammalian homologue of yeast Apg8p, is localized in autophagosome membranes after processing. EMBO J 19: 5720-5728, 2000.

15. Mizushima N, Yamamoto A, Matsui M, Yoshimori T and Ohsumi Y: In vivo analysis of autophagy in response to nutrient starvation using transgenic mice expressing a fluorescent autophagosome marker. Mol Biol Cell 15: 1101-1111, 2004.
16. Liang XH, Jackson S, Seaman M, Brown K, Kempkes B, Hibshoosh $\mathrm{H}$ and Levine B: Induction of autophagy and inhibition of tumorigenesis by beclin 1. Nature 402: 672-676, 1999.

17. Aita VM, Liang XH, Murty VV et al: Cloning and genomic organization of beclin 1, a candidate tumor suppressor gene on chromosome 17q21. Genomics 59: 59-65, 1999.

18. Kato K, Ogura T, Kishimoto A, Minegishi Y, Nakajima N, Miyazaki M and Esumi $\mathrm{H}$ : Critical roles of AMP-activated protein kinase in constitutive tolerance of cancer cells to nutrient deprivation and tumor formation. Oncogene 21: 6082-6090, 2002.

19. Pouyssegur J, Dayan F and Mazure NM: Hypoxia signalling in cancer and approaches to enforce tumour regression. Nature 441: 437-443, 2006.

20. Degenhardt K, Mathew R, Beaudoin B et al: Autophagy promotes tumor cell survival and restricts necrosis, inflammation, and tumorigenesis. Cancer Cell 10: 51-64, 2006.

21. Cuervo AM: Autophagy: in sickness and in health. Trends Cell Biol 14: 70-77, 2004.

22. Amaravadi RK, Yu D, Lum JJ et al: Autophagy inhibition enhances therapy-induced apoptosis in a Myc-induced model of lymphoma. J Clin Invest 117: 326-336, 2007.

23. Paglin S, Hollister T, Delohery T, et al: A novel response of cancer cells to radiation involves autophagy and formation of acidic vesicles. Cancer Res 61: 439-444, 2001.

24. Sobin LH and Wittekind C (eds): TNM: Classification of Malignant Tumors. 6th edition, Wiley-Liss, New York, 2002.

25. Lu Z, Dono K, Gotoh K, et al: Participation of autophagy in the degeneration process of rat hepatocytes after transplantation following prolonged cold preservation. Arch Histol Cytol 68: 71-80, 2005 .

26. Miyata H, Doki Y, Shiozaki H, et al: CDC25B and p53 are independently implicated in radiation sensitivity for human esophageal cancers. Clin Cancer Res 6: 4859-4865, 2000.

27. Yamamoto H, Kondo M, Nakamori S, et al: JTE-522, a cyclooxygenase-2 inhibitor, is an effective chemopreventive agent against rat experimental liver fibrosis. Gastroenterology 125: 556-571, 2003

28. Beasley NJ, Wykoff CC, Watson PH, et al: Carbonic anhydrase IX, an endogenous hypoxia marker, expression in head and neck squamous cell carcinoma and its relationship to hypoxia, necrosis, and microvessel density. Cancer Res 61: 5262-5267, 2001.

29. Ashford TP and Porter KR: Cytoplasmic components in hepatic cell lysosomes. J Cell Biol 12: 198-202, 1962.

30. Mizushima N, Yamamoto A, Hatano M, et al: Dissection of autophagosome formation using Apg5-deficient mouse embryonic stem cells. J Cell Biol 152: 657-668, 2001.

31. Mizushima N: Methods for monitoring autophagy. Int J Biochem Cell Biol 36: 2491-2502, 2004.

32. Jin S and White E: Role of autophagy in cancer: management of metabolic stress. Autophagy 3: 28-31, 2007.

33. Levine B: Cell biology: autophagy and cancer. Nature 446: 745-747, 2007.

34. Alva AS, Gultekin SH and Baehrecke EH: Autophagy in human tumors: cell survival or death? Cell Death Differ 11: 1046-1048, 2004.

35. Ogier-Denis E and Codogno P: Autophagy: a barrier or an adaptive response to cancer. Biochim Biophys Acta 1603: 113-128, 2003.

36. Kondo Y, Kanzawa T, Sawaya R and Kondo S: The role of autophagy in cancer development and response to therapy. Nat Rev Cancer 5: 726-734, 2005.

37. Blommaart EF, Luiken JJ and Meijer AJ: Autophagic proteolysis: control and specificity. Histochem J 29: 365-385, 1997.

38. Martinet W, De Meyer GR, Andries L, Herman AG and Kockx MM: In situ detection of starvation-induced autophagy. J Histochem Cytochem 54: 85-96, 2006.

39. Edinger AL and Thompson CB: Defective autophagy leads to cancer. Cancer Cell 4: 422-424, 2003.

40. Uchiyama Y: Autophagic cell death and its execution by lysosomal cathepsins. Arch Histol Cytol 64: 233-246, 2001.

41. Levine B: Eating oneself and uninvited guests: autophagyrelated pathways in cellular defense. Cell 120: 159-162, 2005.

42. Inbal B, Bialik S, Sabanay I, Shani G and Kimchi A: DAP kinase and DRP-1 mediate membrane blebbing and the formation of autophagic vesicles during programmed cell death. J Cell Biol 157: 455-468, 2002.

43. Qu X, Zou Z, Sun Q, et al: Autophagy gene-dependent clearance of apoptotic cells during embryonic development. Cell 128: 931-946, 2007. 